\title{
Data Aggregation Scheme Using Multiple Mobile Agents in Wireless Sensor Network
}

\author{
Mohamed Younis Mohamed Alzarroug, Wilson Jeberson
}

\begin{abstract}
Wireless Sensor Nodes (WSN) has restricted sensing, communication and computational capabilities, in addition, are mainly operated by means of batteries in a bad atmosphere with the non-replenish-able power sources. As Data aggregation (DA) has more significance in solving the chief limitations of utilizing WSNs, say, the restricted battery life of the powered sensors in addition to short-communication gamut of sensors, it becomes an active research domain today. Effectively gathering data has constantly been the principal significance in WSNs. Regarding the static sink, nodes next to the sink would encompass more loads for routing data, and consequently Mobile Agent (MA) has been commenced. At the moment, the MA could move itself to the sensor nodes (SN) for amassing the data. This MA has made the gathering and aggregation of data possible in a means that is suitable for instantaneous applications. This work proposes an effective DA Scheme in WSN that employs manifold MAs for aggregating data in addition to transferring it to the sink centred on Itinerary planning. This could well be attained by grouping the nodes in clusters as well as planning itineraries effectually amongst cluster heads (CHs) alone. In the proposed DA scheme, itinerary planning is performed utilizing Hybrid Ant Colony Optimization-Genetic Algorithm (ACO-GA). Ultimately, the sink sends the MAs for amassing data as of the $\mathrm{CH}$. Simulation outcome confirms clearly that the proposed work shows high-level performance than the other traditional techniques.
\end{abstract}

Index terms: Wireless Sensor Network, Data Aggregation, Mobile Agents, Cluster Head, Ant Colony Optimization, Genetic Algorithm, and Itinerary Planning.

\section{INTRODUCTION}

The development of a tiny, lower price in addition to low power SNs is possible with the current technological advancements in microelectromechanical systems (MEMS) and also in wire-less communications. WSNs comprise lots of SN compactly placed in the monitoring region with sensing, wire-less communications as well as computing abilities [1]. SN senses the incident for the particular data and transports the outcomes to the sink. The sink is situated adjacent to the sensing area; in addition, it stocks all the forwarded outcomes. WSN with IoT offers an active network infrastructure aimed at DA, distribution, along with processing. It endeavors to unite several packets as of the $\mathrm{SN}$ in an effectual manner in addition to compress the data [2].

Revised Version Manuscript Received on 10, September 2019.

Mohamed Younis Mohamed Alzarroug, Research Scholar, Department of Computer Science \& I.T, Sam Higginbottom University of Agriculture Technology and Sciences (SHUATS), Uttar Pradesh, India. (Email ID: moh_you83@yahoo.com)

Wilson Jeberson, Professor and Head of the Department of Computer Science \& I.T, Sam Higginbottom University of Agriculture, Technology and Sciences (SHUATS), Uttar Pradesh, India. (Email ID: jeberson_w@shiats.edu.in)
A wire-less SN is formed of four practical modules: a) sensing unit, b) transceiver, c) processing unit, and d) power unit. The incessant analog signal as of sensors is transmuted into digitized signal and then sent it to controllers through the analogs-to-digital converters intended for processing [3]. WSN competent to do ' 3 ' fundamental tasks, explicitly,

- Observing environment's conditions

- Processing as well as storing the data for a moment

- Transporting data to user-end via the base station (BS)

WSNs are gradually gaining more importance in everyday lives via joining the physical to the digital world on account of their benefit of simple deployment, fast communication and also low upkeep [4]. The communicational cost in WSN is most of the time above the computational one. For optimizing the communicational cost, DA in WSN is worked out in the access point or sink/CH [5]. DA method could be utilized for aggregating the sensor readings. These readings taken as of a variety of nodes are input to an aggregation algorithm and aggregated data is the output data. Additionally, an effective path is chosen for transferring the aggregated data to a sink node [6]. The '2' schemes of DA are: client-server (CS) and MA. CS permits the source nodes (SN) to transfer their data to a sink or intermediary aggregators for DA. MA data aggregation transfers a single or manifold MA(s) all through a network to gather in addition to aggregate the data samples as of the SN [7].

WSNs have turned into the most well-liked research fields owing to its extensive gamut of developing applications, for instance, habitat monitoring, forest surveillance, and healthcare system. On account of the restriction of cost (specifically hardware), abundant limits arise while planning a WSN, for example transmission range, memory, energy, and also computing power [8]. Interactions with the world are done by means of the sink that is typically joined to a monitoring service via longer-range communication technology (WiMAX, Wi-Fi, satellite communication, et cetera.) [9]. On the off-chance that the sink is static, then the nodes next to the sink expire first since they are utilized every time whilst forwarding data, and it concretes the intricacy of "Energy Hole". On account of that the sink is not capable of getting more data. Thus, researchers embrace sink mobility to lessen the power consumption. [10].

MAs migrate as of node to node utilizing the nodes' resources. The MA-centred model has several advantages such as a diminution in network load, triumphing over the

\section{Published By:}

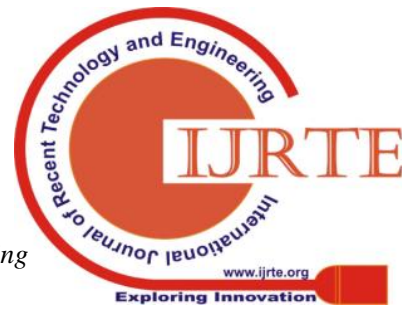


issue of robustness, network latency, as well as fault-tolerance [11]. The Mobile Sink nodes drift along the complete WSN. Furthermore, amass data as of nodes lie down their elected path. Battery is recharged with the addition of mobility in sinks and therefore Energy Consumption (EC) via such nodes is not a matter to them [12]. The DA approach transfers its focus as of discovering the smallest routes to determining routes that let data to be completely grouped. The MA blends the information of every SN to attain a particular accuracy level. For augmenting the protocol's efficiency, manifold MAs might be utilized in place of a single MA [13].

The paper is exhibited as Section 2 asses the literature works with reference to the proposed work. In sections 3 , a succinct discussion concerning the proposed work is offered, section 4 analysis the experiential outcome and also section 5 infers the paper.

\section{LITERATURE SURVEY}

Shengchao $\mathrm{Su}$ and Shuguang Zhao [14] suggested a framework of virtual forces-centric DA with a mobile sink on WSN. The optimum moving trajectories of sink nodes could be attained to effectually save energy and to acquire high efficacy. Lastly, the DA tree was dynamically built for gathering data. This sink node deployed the disseminated path tree for gathering information about neighbor nodes. Lastly, the recommended mechanism attained finer performance on considering the existent methodologies.

Selvakumar Sasirekha and Sankaranarayanan Swamynathan [15] propounded a routing algorithm termed CCMAR (Cluster-Chain MA Routing). It integrated the merits of existent LEACH and also PEGASIS protocols for lessening the transmission delay and EC, respectively. A simulation was done in respect of metrics like EC, network's lifespan and transmission delay. Lastly, the results corroborated that the propounded CCMAR approach outperformed the existent aforesaid protocols centered on the considered metrics.

R. Yuvaraj et.al [16] proffered a time-oriented LEAD (Location Energy Availability Data Rates) centric scheduling algorithms with the intention of improving the data collection performance of WSN. It could schedule sensors for a polling point grounded on the location and energy of sensors. By utilizing NS2 outcomes, it was evinced that TLEAD out-performed MSPR by $46.00 \%, 16.00 \%, 50.00 \%$ and $7.00 \%$ concerning the delay, delivery ratio (DR), packet drops and also residual energy (RE), respectively as the number of nodes $(\mathrm{N})$ was elevated.

L. Sudha and P. Thangaraj [17] recommended an approach that broadly grouped into Node Switching MHL (Multi-Hop LEACH) which exploited the mean randomization of the network energy. Here, the nodes with high-average energy were selected. As of the outcomes, the average end-end delay of Load Balancing Inter-Clusters Network Switching MHL was well-performed via lowering the delay to $10.530 \%$ and $5.410 \%$ than MHL and Node Switching MHL, respectively at $\mathrm{N}=60$ was proved.

Hanady M. Abdulsalam et.al [18] concerned the issue of elevating the WSN lifetime utilizing a cluster-centric DA algorithm. A method was suggested in trouncing the problem.

Mobile Elements (ME) was utilized in the Internet of Things (IoTs) to perform as $\mathrm{CH}$ in a cluster-centric aggregation algorithm. The outcomes evinced that their suggested solution was effectual in respect of saving the sensors' energy and extending the network lifespan. As of the outcomes, the effectual response of the algorithm to the non-existence of MEs and its assurance that data was not misplaced or lost were found.

Amanjot Singh Toor and A.K. Jain [19] propounded a concept termed MEACBM (Mobile Energy Aware Clusters-centric Multi-hop) for the hierarchic-heterogeneous WSNs which picked $\mathrm{CHs}$ grounded on the suggested probability equation. The outcome showed pre-eminent performance via lessening the amounts of EC of SN on a classical manner and thereby elevated the network lifespan, throughput (i.e. packets transferred to the BSs) and number of deceased SNs per cycle in all scenarios that were regarded in simulation.

\section{DATA AGGREGATION SCHEME IN WSN USING MULTIPLE MOBILE AGENTS}

In WSN, numerous sensors are implemented and they produce a massive quantity of data that needed to be delivered to the BS for processing. As the sensors are energy restraint naturally, it is inept to pass on the entire sensor-generated data to the BS. There is consequently a requirement for a technique to combine the data into valuable information at the intermediary nodes, thus lessening the total packets to be moved to a BS. DA is typically the most well-known method to manage these problems, which serves as a clever means of combining as well as compressing the data. Aimed at this purpose, the most normally utilized computing paradigm is $\mathrm{CS}$, wherein every SN communicates its gathered data to a sink by means of the multi-hop route. However, this conventional paradigm endures as of considerable downside, for instance, be short of scalability when the network size augments. In the previous few years, an effective paradigm termed mobile agent paradigm (MA) has appeared and it had been utilized by researchers as an option to the conventional CS paradigm. In this, rather than collecting data and transferring it to a sink via $\mathrm{SNs}$ as in the instance of conventional CS, the mobile node travels to $\mathrm{SN}$ to gather data. The proposed work could be expounded in detail utilizing the block diagram displayed in Figure 1.

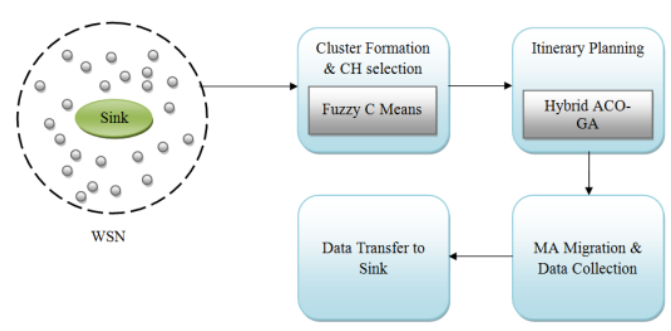

Figure 1: The Proposed Framework 
This paper works on an effective DA Scheme in WSN which utilizes multiple MAs for aggregating the data and transferring it to the sink centred on Itinerary planning. This could be attained by means of grouping nodes on clusters and also planning itineraries effectively amongst $\mathrm{CHs}$ merely. Initially, the $\mathrm{SN}$ is initialized in the WSN in association with multiple MAs. At present, clusters are generated and $\mathrm{CHs}$ are chosen with the aid of Fuzzy C Means (FCM) algorithm. Subsequent to building clusters and choosing $\mathrm{CHs}$, the nodes on the sink are selected to be the 1 st nodes on every branch stemming as of the sink. Every branch gives a route, therefore the number of route would be like that of the branches stemming as of the sink. In the proposed DA scheme, itinerary planning is performed utilizing hybrid-ACO-GA. At last, the sink transmits the MAs to amass data as of the $\mathrm{CHs}$.

\subsection{Initialization of Sensor Nodes in WSN}

During node initialization, the SN in addition to Sink is placed on the WSN. WSN comprises a big number of SN and a sink. The entire $\mathrm{SN}$ are split into '2' categories. One is the common nodes and the other is $\mathrm{CH}$. The common nodes monitor environment information and transmit sensing data to a $\mathrm{CH}$. The SNs in addition to the sink encompass the same maximal transmission gamut; however, with regard to battery power as well as computational abilities, the sink is more influential when weighed against the rest of the SNs. Moreover, the sink is situated at the mid of the monitoring region. In addition, all the SNs encompass the same quantity of energy. The proposed method utilizes manifold MAs which migrate to the entire $\mathrm{CHs}$ and amasses data as of those $\mathrm{CHs}$ of the entire clusters and also transmits it to a Sink.

\subsection{Cluster Formation and CH Selection}

Clustering could be stated as the separation of the nodes within the groups on account of some mechanism. Clustering was revealed to enhance network life span, a prime metric for estimating the sensor network's performance. Clustering is performed to acquire energy efficiency along with the network's scalability. The proposed method uses FCM for the clustering of SN. FCM algorithm produces numerous clusters and for every cluster, a $\mathrm{CH}$ is selected. The $\mathrm{SN}$ with the highest $\mathrm{RE}$ is picked as the $\mathrm{CH}$.

\subsubsection{Cluster formation}

In the proposed method, the FCM is embraced for the formation of cluster. This FCM clustering protocol stands as a centralized clustering mechanism. The BS works out as well as allots $\mathrm{SN}$ to clusters as per its geographical location information. The $\mathrm{CH}$ is allotted to the node bearing the maximal RE inside the cluster. A network of $\mathrm{SN}$ that is separated into clusters is concerned. Here, every node is allotted with a degree of member-ship to a cluster instead of entirely being a member of merely ' 1 ' cluster. Thus, the nodes near the boundary of a cluster might turn out to be a member of the cluster with the approximate degree of member-ship to a neighboring cluster. The cluster formation is executed to lessen the objective function.

$$
F_{m}=\sum_{u=1}^{c} \sum_{v=1}^{N} \mu_{u v}^{m} d_{u v}^{2}, \quad 1 \leq m<\infty
$$

Where, $m$ is the fuzzy field parameter and is basically a real number of more than ' 1 ', $\mu_{u v}$ implies the degree of member-ship, $d_{u v}$ signifies the Euclidean distance (ED) between cluster center $\left(c_{v}\right)$ and node.

The distance betwixt the $\mathrm{SN}$ and centre point is taken as the ED. By attaining the spatial distance's minimization, the allotment of nodes to the clusters is optimized; therefore energy balance could be attained.

Subsequently, fuzzy partitioning is performed via the iterative optimization of an aforesaid objective function, with the cluster centres $c_{v}$ and the update of member-ship $\mu_{u v}$.

$$
\begin{gathered}
c_{v}=\frac{\sum_{u=1}^{N} \mu_{u v}^{m} * d_{u}}{\sum_{u=1}^{N} \mu_{u v}^{m}} \\
\mu_{u v}=\frac{1}{\sum_{w=1}^{C}\left(\frac{\left\|d_{u v}\right\|}{\left\|d_{w v}\right\|}\right)^{2 / m-1}}
\end{gathered}
$$

This iteration will end when it fulfils the condition given in equation (4),

$$
\max _{u v}\left\{\left|\mu_{u v}^{(w+1)}-\mu_{u v}^{(w)}\right|\right\}<\epsilon
$$

Wherein, $\in$ implies a termination criterion betwixt zero and one, while $w$ are the iteration steps. This converges to a local minimal or a saddle point of $F_{m}$. The Pseudocode for forming clusters utilizing FCM is exhibited in Figure 2.

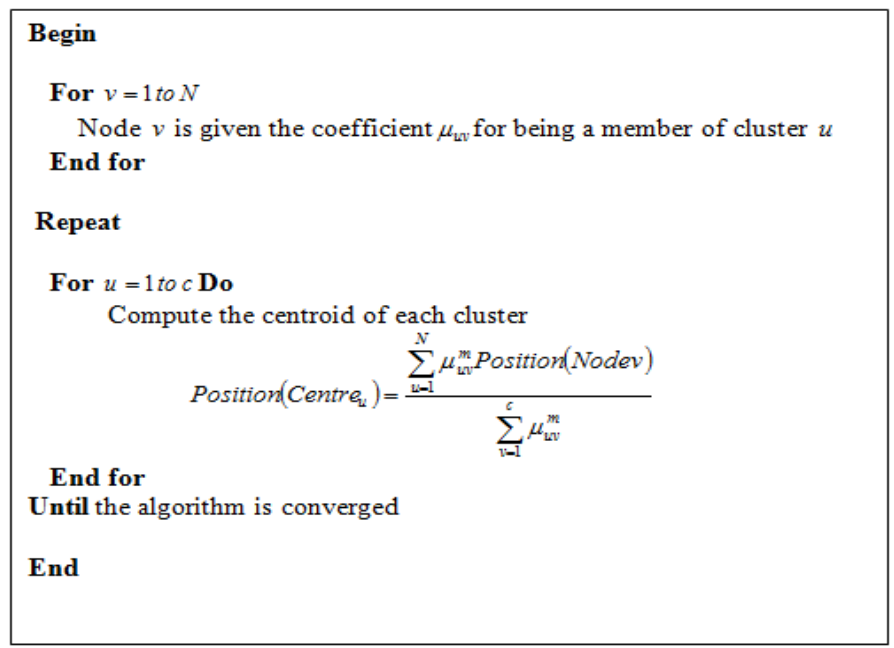

Figure 2: Pseudocode of FCM for Cluster Formation

\subsubsection{Cluster head selection}

Subsequent to forming the clusters, the sink selects a node encompassing the highest RE amid every cluster as the $\mathrm{CH}$. After that an assignment message is dispatched to each $\mathrm{SN}$ on the network encompassing the cluster's information it fits in to and the time timetable to transmit the data. After the assignment message attains an $\mathrm{SN}$, the node extorts the 
network details as of this message, for instance, the $\mathrm{CH}$ identification in addition to transmission time schedule, in addition, stocks this details in its memory for forwarding data amid the data transmission stage. The cluster formation along with $\mathrm{CH}$ selection recurs at regular intervals amid the network process with the time cycle pre-stated while installing the $\mathrm{SN}$ firmware.

\subsection{Itinerary Planning Based on $\mathrm{CH}$}

Subsequent to building clusters and choosing $\mathrm{CHs}$, the nodes on the sink are selected as the 1st nodes on every branch stemming as of the sink. Every branch gives an itinerary, consequently, the total itinerary would be like that of the number of branches stemming as of the sink. For generating the itinerary, itineraries are designed for MAs amongst CHs by utilizing hybrid ACO algorithms. The proposed algorithm utilizes a joint ACO and GA algorithms to set up the itinerary planning betwixt the $\mathrm{CH}$ nodes and the sink via multiple MAs.

\subsubsection{ACO phase}

The steps in the Ant Colony Optimization (ACO) of the ACO-GA method are enumerated and expounded below.

\section{Parameter Setting:}

Initially, $R$ numbers of artificial ants are produced at every number of iteration. The $R$ value is selected centred upon the network topology's size. Every link is related to an exceptional variable termed a pheromone that could also be comprehended and even customized through the ants. The pheromone value ' $\tau_{a b}$, placed in the $c_{a b}$, is related to the solution component ${ }^{s_{a b}}$. The collection of the entire probable solution components is implied by means of $S$. Moreover, the number of pheromone variables is centred upon the number of quality metrical taken in favour of route selection.

\section{Objective Function:}

The rules for the selection of the solution components are specified,

- The cost, i.e., minimum delay in addition to minimum hops, of the chosen route ought to be minimal

- The chosen route ought to be an existing link.

- The path ought to meet transmission restraints.

An objective function is evaluated as,

$$
O F=\frac{1}{\operatorname{Cost}(d)}
$$

$\operatorname{Cost}(d)$ regards two additive QoS parameters. On the off-chance that an ant is existent on the node ${ }_{b}$, then the subsequent node $G_{b}$ is stochastically chosen amongst the previous un-visited nodes. Specially, the un-visited trail is chosen with the probability which is proportional to the pheromone related to the $\operatorname{link}^{c_{a b}}$.

\section{Path Selection:}

If the ant ' $k$, is at $G_{a}$, then the subsequent node $G_{b}$ is picked in line with the equ (6).

$$
P_{a b}^{k}=\frac{\left(\tau_{a b}^{\alpha}\right)\left(\eta_{a b}^{\beta}\right)\left(D_{a b}\right)^{\gamma}}{\sum_{c_{i l \in H\left(F^{P}\right)}\left(\tau_{a b}^{\alpha}\right)\left(\eta_{a b}^{\beta}\right)\left(D_{a b}\right)^{\gamma}}}
$$

Here, $P_{a b}$ indicates the probability with which the ant $k$ chooses edge $e_{a b}, H\left(F^{P}\right)_{\text {implies the collection of feasible }}$ components, The $\left(D_{a b}\right)^{\gamma}$ signifies the relative metric for delay as of $a$ to ${ }^{b} . \alpha$ and $\beta$ implies control parameters with the relative implication of the pheromone. Heuristic information ، $\eta_{a b}$ ' is provided via the equ (7).

$$
\eta_{a b}=\frac{1}{d_{a b}}
$$

Local Update and Global Update:

The pheromone's local update is done by the entire ants subsequent to every step. Every ant implements it merely to the final edge traversed. The pheromone is updated utilizing the equation (8) when ant effectively completes a hop as of to $\tau_{a b}=(1-\rho) \cdot \tau_{a b}+\sum_{k=1}^{u} \Delta \tau_{a b}^{k} \quad, 0 \prec \rho \prec 1$

Wherein,

$\rho$ indicates the residual pheromone co-efficient, $(1-\rho)$ signifies the pheromone evaporation rates, $u$ implies the total number of ants as well as $\Delta \tau_{a b}^{k}$ symbolizes the pheromone value placed via $k^{\text {th }}$ ant whilst passing via ${ }_{a b}$.

The global update of pheromone after construction is then given using the equation (9).

$$
\tau_{a b} \leftarrow(1-\rho) \cdot \tau_{a b}+\rho \cdot \Delta \tau_{a b}^{b e s t}
$$

Where,

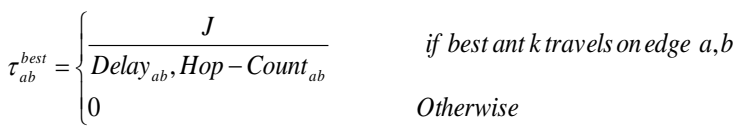

Here, $J$ is a constant, Delay ${ }_{a b}$ indicates the relative metrics intended for the end-end delay, whereas, Hop - Count ${ }_{a b}$ signifies the number of hops betwixt source and destination.

\section{Termination:}

Next, the solution set encompasses the available routes as of any source-destination which fulfils the minimal QoS requirements. On the off-chance that the maximal number of iterations is attained, then ACO gives the solution set to the following phase i.e., GA.

\subsubsection{GA phase}

The resultant path set with premium pheromone computed by means of the ants in above phase (ACO) is regarded as the preliminary populace for this phase. This would eradicate the weaker paths as of the set and hold the top-fit paths centred upon the fitness function and implemented genetic operations. After this, an optimal path set that comprises the needed QoS parameters and helpful for multi-path routing is attained. The steps in the GA of the ACO-GA technique are listed below.

The flowchart for the Hybrid ACO-GA is depicted in Figure 3.

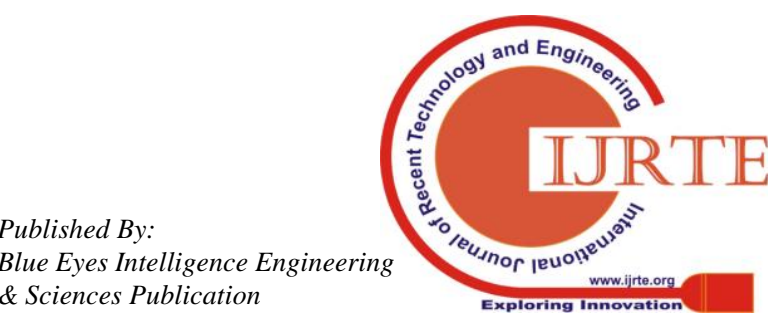




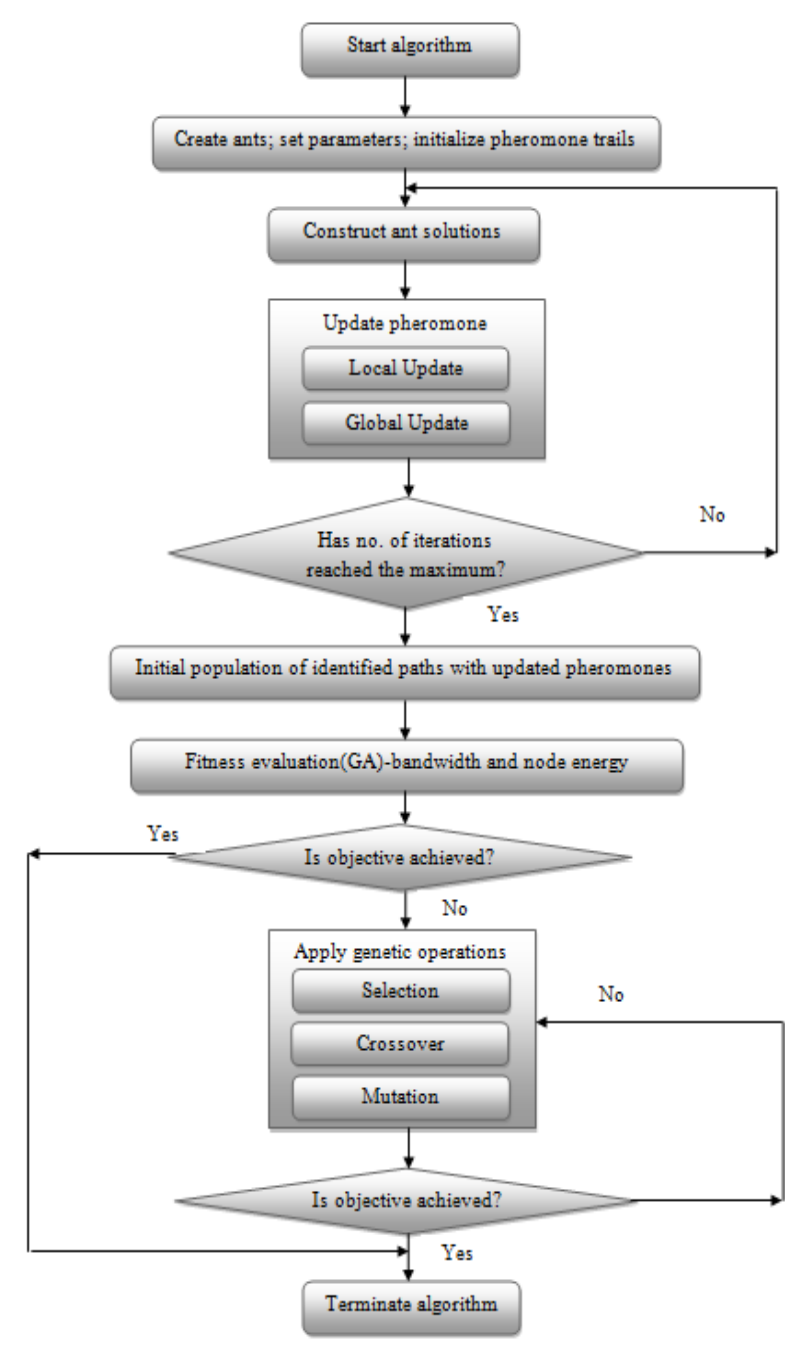

Figure 3: Flowchart of Hybrid ACO-GA

\section{Population Initialization:}

The manifold paths are encoded stochastically via the network of artificial ants with the pheromones so as to attain the initial populace. For such encoding, every single node series of a path called an individual is taken as a chromosome. A node on the recognized network path is therefore taken as a gene. The optimal populace size is also ascertained. Just like the number of hops betwixt source-destination could differ, chromosome length could also be differed. It is utilized to confine the number of paths as of source to destination on the off-chance that there are plenty of them fulfilling the minimum fitness. Centred upon the fitness evaluation as well as ranking, just the high-rated paths are kept.

\section{Selection Operation:}

It is stochastic. It probabilistically chooses the good solutions and eliminates the bad ones centred upon the evaluation made with objective function. A roulette wheel process is applied, in which each path $a$ is allotted a probability ${ }^{p_{a}}$ with which they are selected for reproduction. Subsequent to reproduction, the cumulative probability ${ }^{x_{a}}$ is evaluated for each. A path is picked only if $x_{a}>r$ where, $r$ signifies a random number chosen a priori. ${ }^{x_{a}}$ is evaluated by the equation (11).

$$
x_{a}=\sum_{b=1} p_{b}
$$

\section{Crossover Operation:}

Crossover, a genetic re-combination process, arbitrarily chooses a collection of nodes as of every valid path for generating a new best path. Consider paths along with to be arbitrarily selected and also consider that the nodes in each are recognized. Amongst the similar nodes, a single gene or a gene pattern is detected and then swapped betwixt the nodes for crossover. A single point cross-over approach is implemented for a single node, i.e., as of that node forward, the packet goes on a disparate path. For a node pattern, a two-point cross-over approach is applied, i.e., the data goes on a disparate sub-set of the path. The cross-over pattern is ascertained centred upon the chromosomal length (path length). Provided that, no common nodes are existent betwixt the ' 2 ' arbitrarily picked paths, and then it would pick another collection of paths.

\section{Mutation Operation:}

Mutation comprises arbitrarily changing one or more fractions of a chromosome, which is performed via altering an arbitrarily selected node as of an arbitrarily picked path into another node . is recognized as of the neighbour node set . This substitution is centred on and it averts the establishment of unavailable path. On the off-chance that there is no right node on for substitution, then this might be ignored.

\section{Termination:}

For every populace, the GA operators are implemented to the chromosomes for leading it to a new generation $(\mathrm{GN})$ of individuals. Subsequent to a fixed number of GN, where $G N_{\text {min }} / G N_{\max }$ implies minimal and maximal iterations are attained or when the best fitness value is not any more enhanced as of one to the subsequent GN, in addition no exclusive offspring is incorporated in the new populace for three consecutive times, the process is ended.

Finally, a set of the itinerary is planned for the MAs to reach the $\mathrm{CH}$ of all the clusters.

\subsection{Migration of Multiple MAs for Data Collection}

Subsequent to the organization of the network on clusters and establishment of the itineraries amongst $\mathrm{CH}$, the sink sent MA for accumulating data as of a $\mathrm{CH}$. Initially, when MA meet the $\mathrm{CH}$ for the 1st point in time, it signals the nodes inside the gamut of the $\mathrm{CH}$ to transmit the gathered data to the chosen $\mathrm{CH}$, and subsequently when the MA turn up at the final $\mathrm{CH}$ on the itinerary, it begins gathering the data as of the $\mathrm{CH}$ while coming back to the sink. Such wise, MA uses less time and energy on data collection.

\section{RESULT AND DISCUSSION}

The investigational outcomes for the proposed DA scheme are evaluated in this section. The performance proffered by the proposed DA is assessed by means of Itinerary Planning 
(DAS-IP) using combined ACO-GA. This assessment is performed utilizing Network Simulator 2. The proposed work is compared using the existent technique named as dynamic MA-based data aggregation approach (DMA-DA). The metrics utilized for evaluation are Delay, Energy, DR, Drop, Throughput and Overhead. The simulated WSN that depicts the clusters, cluster member nodes, $\mathrm{CH}$ nodes, MAs and sink node are displayed in Figure 4

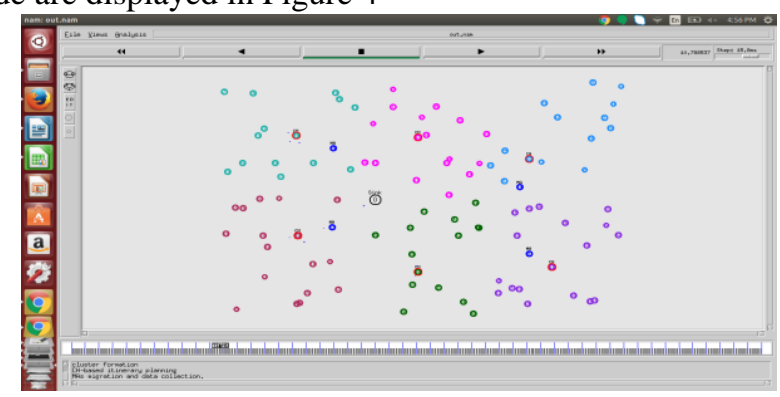

Figure 4: Simulated WSN

The simulation parameters that are exercised in the proposed technique are enlisted in Table 1.

\begin{tabular}{|c|c|}
\hline Parameters & Values \\
\hline Number of Nodes & $20,40,60,80,100$ \\
\hline Topology Area & $1000 \mathrm{~m} \times 1000 \mathrm{~m}$ \\
\hline Routing Protocol & AODV \\
\hline MAC TYPE & $\mathrm{MAC} / 802 \_11$ \\
\hline Propagation & Two-Ray Ground \\
\hline Antenna & Omni-Antenna \\
\hline Simulation Time & 50 seconds \\
\hline Traffic Type & CBR \\
\hline Packet Size & 512 bytes \\
\hline Rate & $100 \mathrm{kbps}$ \\
\hline Channel Bandwidth & $2.0 \mathrm{e} 6$ \\
\hline Initial Sending Power & 0.660 \\
\hline Initial Receiving Power & 0.395 \\
\hline Initial Idle Power & 0.035 \\
\hline Initial Energy & 10.3 Joules \\
\hline Channel Frequency $(\mathrm{Hz})$ & freq $2.4 \mathrm{e} 9$ \\
\hline Transmitter Signal Power (Watt) & Pt_0.28 \\
\hline Mobility Speed & $2-20 \mathrm{~m} / \mathrm{s}$ \\
\hline
\end{tabular}

Table 1: Simulation Parameters

Table 2: Comparison of the proposed DAS-IP and the existing DMA-DA in terms of metrics such as Delay, DR, and Drop

\begin{tabular}{|c|c|l|c|l|c|c|}
\hline Metrics & \multicolumn{2}{|c|}{ Delay } & \multicolumn{2}{c|}{ Delivery Ratio } & \multicolumn{2}{c|}{ Drop } \\
\hline $\begin{array}{l}\text { Number } \\
\text { of Nodes } \\
\text { (N) }\end{array}$ & Proposed & $\begin{array}{l}\text { Existing } \\
\text { DMA-DA }\end{array}$ & Proposed & $\begin{array}{l}\text { Existing } \\
\text { DMA-DA }\end{array}$ & Proposed & $\begin{array}{l}\text { Existing } \\
\text { DMA-DA }\end{array}$ \\
\hline $\mathbf{1 0 0}$ & 7.38156 & 15.308102 & 0.709117 & 0.386867 & 6 & 25 \\
\hline $\mathbf{2 0 0}$ & 11.28197 & 17.303762 & 0.615861 & 0.272461 & 9 & 17 \\
\hline $\mathbf{3 0 0}$ & 15.00316 & 17.269328 & 0.382883 & 0.172134 & 24 & 26 \\
\hline $\mathbf{4 0 0}$ & 20.277569 & 22.16062 & 0.186749 & 0.078482 & 40 & 378 \\
\hline $\mathbf{5 0 0}$ & 20.409008 & 25.571875 & 0.141381 & 0.058461 & 158 & 1034 \\
\hline
\end{tabular}

Table 2 analyzes the proposed DAS-IP and the existing proposed technique shows improved results contrasted with DMA-DA's performance in respects of metrics, for instance, the existing one.

Delay, DR, and Drop. It could be clearly viewed that the

Table 3: Comparison of the proposed DAS-IP and the existing DMA-DA in terms of metrics such as Energy,

Overhead and Throughput

\begin{tabular}{|c|c|c|c|c|c|c|}
\hline Metrics & \multicolumn{2}{|c|}{ Energy } & \multicolumn{2}{c|}{ Overhead } & \multicolumn{2}{c|}{ Throughput } \\
\hline $\begin{array}{c}\text { Number of } \\
\text { Nodes (N) }\end{array}$ & Proposed & $\begin{array}{c}\text { Existing } \\
\text { DMA-DA }\end{array}$ & Proposed & $\begin{array}{c}\text { Existing } \\
\text { DMA-DA }\end{array}$ & Proposed & $\begin{array}{c}\text { Existing } \\
\text { DMA-DA }\end{array}$ \\
\hline $\mathbf{1 0 0}$ & 6.463972 & 13.61866 & 1909 & 2665 & 13542 & 1031 \\
\hline $\mathbf{2 0 0}$ & 7.80906 & 13.192729 & 1857 & 4775 & 11439 & 1301 \\
\hline $\mathbf{3 0 0}$ & 6.805129 & 12.490482 & 2828 & 6524 & 10831 & 1123 \\
\hline $\mathbf{4 0 0}$ & 6.309804 & 11.40279 & 5426 & 9620 & 10133 & 755 \\
\hline $\mathbf{5 0 0}$ & 5.750889 & 10.741787 & 6685 & 13291 & 9452 & 777 \\
\hline
\end{tabular}

Table 3 analyzes the proposed DAS-IP along with the existing DMA-DA's performance in respects of metrics, for instance, Energy, Overhead and Throughput. It could be clearly perceived as of the table that the proposed technique shows enhanced results when weighed against the existing one.

\subsection{Performance Analysis}

The proposed DAS-IP using combined ACO-GA's performance is analyzed and weighed against the existing technique named as DMA-DA in respect of metrics, for

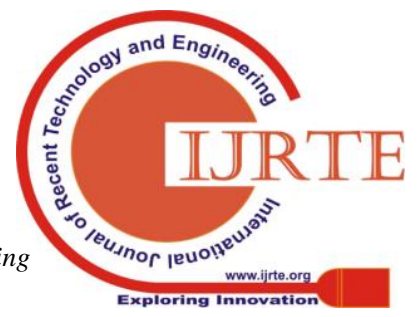


instance, Delay, DR, Drop, Energy, Overhead and Throughput.

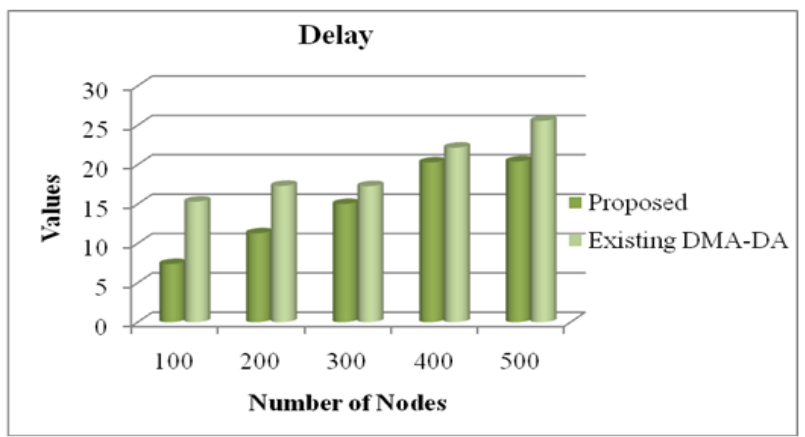

Figure 5: Performance Analysis of the proposed DAS-IP and the existing DMA-DA in terms of Delay

Figure 5 compares the delay against the number of nodes (N) for the existing DMA-DA and the proposed DAS-IP technique. It could well be inferred as of the figure that as the ' $\mathrm{N}$ ' increases, the delay for routing also increases. On considering 500 nodes, the delay is too high for the existent technique. But, the proposed technique's delay varies by 5.162867 values below the existing one. Also, for any ' $N$ ', when weighed against the existing one, the proposed DAS-IP shows less delay for the routing data to the sink.

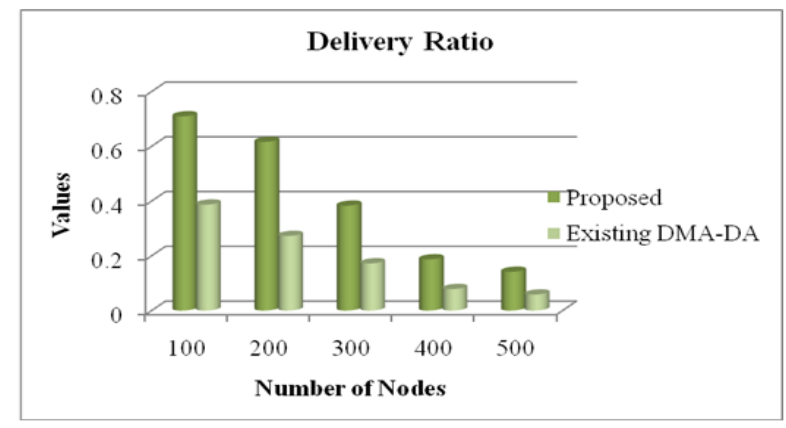

Figure 6: Performance Analysis of the proposed

DAS-IP and the existing DMA-DA in terms of DR

Figure 6 compares the DR against the ' $\mathrm{N}$ ' for the existing DMA-DA and the proposed DAS-IP technique. The data-DR is gauged by the total data received at destinations (Sink) divided by means of the total data sent as of the SN. It can be inferred that the DR decreases as the ' $N$ ' increases. For 100 nodes, the DR is too high for the proposed DAS-IP technique and its value is 0.709117 , however, the DR is lower for the existing one. For higher ones, say 500, the DR decreases compared to the lower ' $N$ '. But, in respects of DR, the proposed work shows improved results.

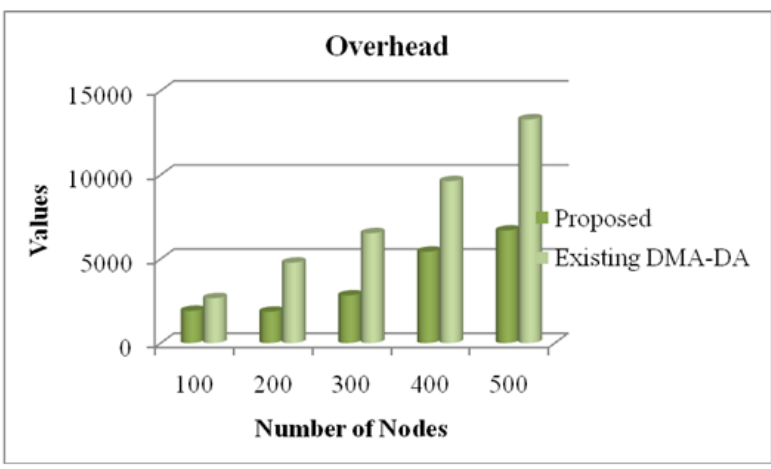

Figure 7: Performance Analysis of the proposed DAS-IP and the existing DMA-DA in terms of Overhead
Figure 7 compares the overhead against the ' $\mathrm{N}$ ' for the existing DMA-DA in addition to the proposed DAS-IP technique. Overhead is linked with the complete number of routing data transmitted amid simulation. It gauges the scalability of a protocol and the degree to which it will function in cramped or lower bandwidth surroundings. It could be perceived as of the graph that, as the ' $N$ ' augments, the overhead for the proposed and the existing also increases. Among the existing and the proposed method, lower overhead is inferred in the proposed technique. Therefore, the proposed one shows higher performance on account of overhead.

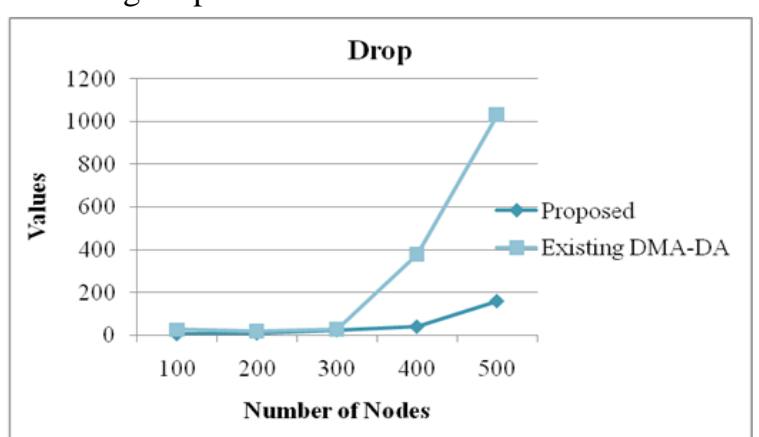

Figure 8: Performance Analysis of the proposed DAS-IP and the existing DMA-DA in terms of Drop

Figure 8 compares the drop against the ' $\mathrm{N}$ ' for the existing DMA-DA and the proposed DAS-IP technique. It could be clearly perceived that the drop value increases for higher ' $N$ '. For 100, 200 and 300 nodes, the drop value remains constant for both the proposed DAS-IP and the existing DMA-DA techniques. But, as the ' $\mathrm{N}$ ' is increased, the drop value also increases. In such cases, the existing technique shows the worst performance, which has the drop value of 1034 for 500 nodes. But the proposed work exhibits a lower drop value compared to the existing one. This confirms the highest performance of the proposed technique.

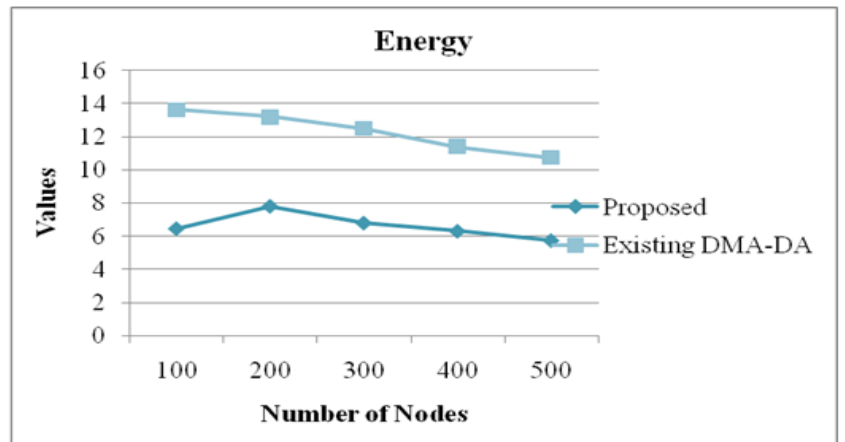

Figure 9: Performance Analysis of the proposed DAS-IP and the existing DMA-DA in terms of Energy

Figure 9 compares the energy against the ' $\mathrm{N}$ ' for the existing DMA-DA and the proposed DAS-IP technique. The whole network energy level could be ascertained by summing every node's energy level in the network. Compared existing technique consumes a vast quantity of energy for any ' $N$ '. But, the proposed technique consumes an almost equal quantity of energy for any ' $\mathrm{N}$ ' and more importantly, the energy consumed is too less when weighed against the existent one.

Published By: 


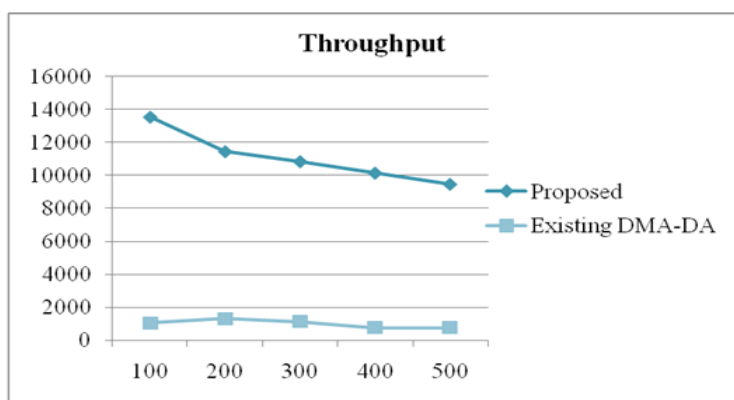

Figure 10: Performance Analysis of the proposed DAS-IP and the existing DMA-DA in terms of Throughput

Figure 10 compares the throughput against the ' $\mathrm{N}$ ' for the existing DMA-DA and the proposed DAS-IP technique. Throughput is basically the total data packets successfully transferred as of a source to a destination in a provided time. For 100 nodes, the throughput is greater for the proposed technique and it reduces as the node increases. But, the throughput of the existent technique is lower for any ' $N$ '. Furthermore, the proposed one's performance is more than the existing one for any ' $\mathrm{N}$ '. This shows the improved performance of the proposed work.

\section{CONCLUSION}

MA data aggregation routing forwards MA in WSN for collecting and aggregating the data. The DA routing targets to elevate the number of gathered data samples and minimize the data collection delay and network resource consumption. This paper proposed an effectual DA Scheme in WSN that utilizes multiple MAs for aggregating and transferring the data to a sink centered on Itinerary planning. This could be attained via grouping the nodes in clusters and effectually planning the itineraries only amongst CHs. The outcomes are evaluated centred on $\mathrm{N}$. The performance comparison is done betwixt the proposed DAS-IP and existent DMA-DA methodology in respect of metrics say, Delay, Overhead, DR, Throughput, Drop, and Energy. The evaluated outcomes confirm the dominance of the proposed technique.

\section{RFERENCES}

1. Mohamed El Fissaoui, Abderrahim Beni-Hssane, and Mostafa Saadi, "Energy efficient and fault tolerant distributed algorithm for data aggregation in wireless sensor networks", Journal of Ambient Intelligence and Humanized Computing, pp. 1-10, 2018.

2. Dnyaneshwar S. Mantri, Neeli Rashmi Prasad, and Ramjee Prasad, "Mobility and heterogeneity aware cluster-based data aggregation for wireless sensor network", Wireless Personal Communications, vol. 86, no. 2, pp. 975-993, 2016.

3. Abirami, T., and S. Anandamurugan, "Data aggregation in wireless sensor network using shuffled frog algorithm", Wireless Personal Communications, vol. 90, no. 2, pp. 537-549, 2016.

4. Sreevidya, B., and M. Rajesh, "Enhanced energy optimized cluster based on demand routing protocol for wireless sensor networks", In 2017 International Conference on Advances in Computing, Communications and Informatics (ICACCI), pp. 2016-2019. IEEE, 2017.

5. Kaustuv Sarangi, and Indrajit Bhattacharya, "A study on data aggregation techniques in wireless sensor network in static and dynamic scenarios", Innovations in Systems and Software
Engineering, pp. 1-14, 2019.

6. Sukhchandan Randhawa, and Sushma Jain, "Data aggregation in wireless sensor networks: Previous research, current status and future directions", Wireless Personal Communications, vol. 97, no. 3, pp. 3355-3425, 2017.

7. Saeid Pourroostaei Ardakani, Julian Padget, and Marina De Vos, "A mobile agent routing protocol for data aggregation in wireless sensor networks", International Journal of Wireless Information Networks, vol. 24, no. 1, pp. 27-41, 2017.

8. Chi-Fu Huang, and Wei-Chen Lin, "Data collection for multiple mobile users in wireless sensor networks", The Journal of Supercomputing, vol. 72, no. 7, pp. 2651-2669, 2016.

9. Abdul Waheed Khan, Javed Iqbal Bangash, Adnan Ahmed, and Abdul Hanan Abdullah, "QDVGDD: Query-driven virtual grid based data dissemination for wireless sensor networks using single mobile sink", Wireless Networks, vol. 25, no. 1 , pp. 241-253, 2019.

10. Rathod Yogirajsinh, Mehul B. Shah, and Parthesh Mankodi, "Efficient data gathering in wireless sensor network using mobile sink", In 2016 IEEE International Conference on Recent Trends in Electronics, Information \& Communication Technology (RTEICT), pp. 1416-1420. IEEE, 2016.

11. Divya Lohani, and Shirshu Varma, "Energy efficient data aggregation in mobile agent based wireless sensor network", Wireless Personal Communications, vol. 89, no. 4, pp. 1165-1176, 2016.

12. Sanjima Rana, and Khushal Thakur, "Extrapolated-grid based Mobile Sink Data path selection for Wireless Sensor Network", In 2015 Annual IEEE India Conference (INDICON), pp. 1-5. IEEE, 2015.

13. Mehak and Padmavati Khandnor, "Energy efficient data aggregation using multiple mobile agents in wireless sensor network", In Smart Innovations in Communication and Computational Sciences, pp. 279-287. Springer, Singapore, 2019.

14. Shengchao $\mathrm{Su}$, and Shuguang Zhao, "A novel virtual force-based data aggregation mechanism with mobile sink in wireless sensor networks", Cluster Computing, pp. 1-16, 2018.

15. Selvakumar Sasirekha, and Sankaranarayanan Swamynathan, "Cluster-chain mobile agent routing algorithm for efficient data aggregation in wireless sensor network", Journal of Communications and Networks, vol. 19, no. 4, pp. 392-401, 2017.

16. Yuvaraj, R., A. Chandrasekar, and S. Jothi, "Time orient LEAD based polling point selection algorithm for efficient data aggregation in wireless sensor networks", Cluster Computing, pp. 1-8, 2018.

17. Sudha, L., and P. Thangaraj, "Improving energy utilization using multi hop data aggregation with node switching in wireless sensor network", Cluster Computing, pp. 1-9, 2018.

18. Hanady M. Abdulsalam, Bader A. Ali, and Eman AlRoumi, "Usage of mobile elements in internet of things environment for data aggregation in wireless sensor networks", Computers \& Electrical Engineering, 2017.

19. Amanjot Singh Toor, and A. K. Jain, "Energy aware cluster based multi-hop energy efficient routing protocol using multiple mobile nodes (MEACBM) in wireless sensor networks", AEU-International Journal of Electronics and Communications, 2019. 